\title{
Complexity and Workload Factors in Virtual Work Environments of Mobile Work
}

\author{
Ursula Hyrkkänen $^{1}$, Ari Putkonen ${ }^{1}$, and Matti Vartiainen ${ }^{2}$ \\ ${ }^{1}$ Turku University of Applied Sciences, Sepänkatu 3, 20700 Turku, Finland \\ ${ }^{2}$ Laboratory of Work Psychology and Leadership, Department of Industrial Engineering and \\ Management, Helsinki University of Technology, P.O.Box 5500, FIN-02015 TKK, Finland \\ ursula . hyrkkanen@turkuamk. fi
}

\begin{abstract}
This article concentrates on describing the complexity and work load factors of mobile work done in virtual environments. A qualitative multi case study was carried out. Six mobile employee groups were examined. The data was collected by interviews and questionnaires. A model of complexity factors was used in analyzing the data. The complexity factors interrelated with different types of workload components, i.e. physical, mental and social and, furthermore, they induced distinct workload factors. To reduce the manifestation of the workload factors and to enhance well-being, fundamental requirements for the virtual environment can be presented. At the levels of connection, device and application the issue lies in the transfer capability of communication. Compared to this at the levels of cognitive and cultural factors of the virtual space the question is in the ability of semantic transfer of the message.
\end{abstract}

Keywords: Mobile work, virtual work environment, work load factor, well-being.

\section{Introduction}

Mobile work and its practices have attracted the attention of researchers from various research disciplines. However, research on mobile work is in its early stages and definitions of mobility are still emerging. A central problem related to developing types of work, such as mobile work, is that its new working procedures and its new working environments - especially the virtual working spaces - are not known well enough. For example, it is difficult to connect employee well-being outcomes to the unknown characteristics of the work. If environmental complexity factors are well managed in a traditional work environment, they really are a relevant issue with mobile work done in virtual environments. Principally, the virtual work environment of the employee is unknown and not controlled by the managers or virtual environment designers. It is hardly self-managed by the employee and this may cause additional challenge and strain.

The purpose of this article is to show the complexity and workload factors of mobile work done in virtual work environments [11]. The work is defined "mobile", if the employee works more than ten hours per week outside of the primary workplace 
and uses information and communication technology (ICT) for communication [6]. The use of ICT tools generates the virtual work environment.

This paper shows the complexity and workload factors related to the different dimensions of mobile work from the perspective of the virtual work environment. The questions to be studied are:

1. How do the complexity factors manifest themselves in mobile work from the perspective of the virtual work environment?

2. What are the dimension-related work load factors of mobile work in virtual work environments?

This article is organized as follows. First, the background section introduces the complexity approach and the main concepts. Next the methodology, methods and research settings of this case study are presented. Thereafter, the article describes the empirical results illustrating the complexity and workload factors of mobile work done in virtual spaces. Finally, the results are summarized and discussed and some future trends are presented in the concluding section.

\section{Definitions of Mobile Work and the Complexity Approach}

The physical mobility of employees is realized at least at two levels: individuals move alone as members of a distributed team or organization and teams and projects move as a part of a dispersed organization or network using different sites. Mobile employees establish their "instant office" by adapting to and using the environment at hand, and do so again as quickly. If collaboration with distant workmates is needed, this is only possible with mobile, wireless information and communication technologies.

First discussions on the concept of mobility dealt with employees moving from place to place. Kakihara and Sørensen [9] described three interrelated aspects of worker mobility: location mobility concerns the workers extensive geographical movement, operational mobility deals with the flexible operations of independent business units and interaction mobility the intense of fluid interactions of actors. Andriessen and Vartiainen [2] expanded the concept to cover also virtual mobility so that it includes stationary actors moving with help of ICT tools in virtual working spaces. Mobile workers are those employees who move a lot and collaborate with others via electrical tools. This article highlights the concept of mobile work from the viewpoints of physical mobility and virtual collaboration.

The complexity factors of the work refer to the wide range challenges, which are inherent in the work and can be examined as its characteristics and demands. The complexity of work is usually considered as a factor related to the task. At one end the task is creative and demanding, and, at the other end, it is simple and routine-like [1]. The expanded complexity concept considers also the working environment that can be a different combination of physical, virtual, social and cultural spaces.

Vartiainen [15] described by six dimensions the complexity of working contexts, which complement the complexity of the task as the two main factors influencing intra-individual and -group processes needed in coping with complexities. They are 
geographical dispersion of the working locations, physical mobility, diversity of actors, asynchronous working time, temporary structure of working and mediated interaction as shown in Figure 1 [10]. These six dimensions form in addition to task complexity a set of requirements that can also considered as workload factors. The workload-strain model draws a distinction between the effects of external sources impinging upon a human being (workload) and the effects (strain) within individuals, depending on their per-existing conditions [13]. The transactional model of stress for it's part states that the interaction between a person and the environment creates a perceived stress on the individual. Stress is not a property of the person, nor the property of the environment, but arises from the conjunction of a particular kind of environment and particular kind of person [12].

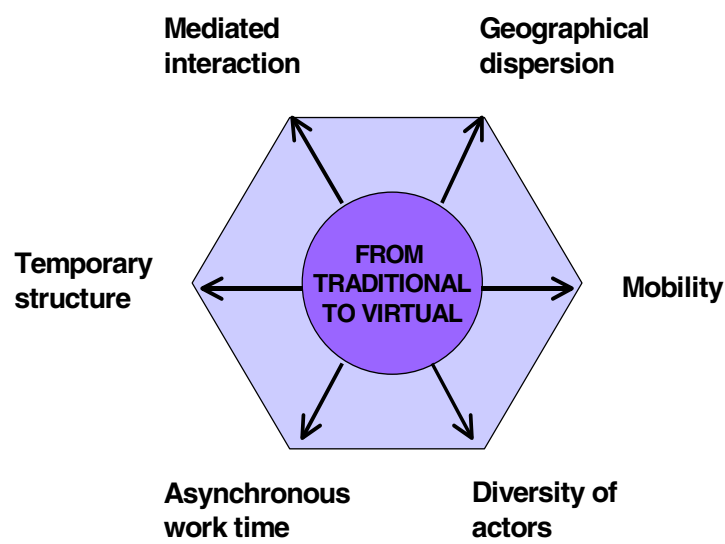

Fig. 1. Complexity factors of working environment [15, pp. 22-23]

According to previous studies [5, 10], geographical dispersion influence on working practices and needs of communication and coordination. It has also been proven that mobile and multi-locational work increases the physical distance of the workers of the main team and hinders the face-to-face communication of the team. On account of this, the need for using wireless technologies increases. Non-verbal cues are usually absent in mediated communication, and this may easily lead to misunderstandings and lack of trust. The global groups in particular have members with different backgrounds, i.e. a different language, culture, values, orientation to work, leadership type, etiquette and punctuality. These may further create communication problems. The temporary nature of their projects leads to loose social engagement due to limited expectations of working together again. Asynchronous work time in relation to the main team makes additional demands on communication.

In this paper, the model of complexity factors [15] is applied to analyse and realize the features of mobile work done in virtual environments. 


\section{Research Method and Data Collection}

The study was carried out as a qualitative multi-case study [16], in which six mobile employee groups were explored; three groups moving globally and three groups moving locally. The number of employees in each group varied between five and eight (altogether 41 employees were met). The data was gathered by interviewing each member of the groups by semi-structured interview. In addition, three different surveys were used: virtual team questionnaire (VTQ1), well-being in dispersed work (WDW) and Job Engagement Survey [14].

A model of the complexity factors [15] was used in analysing the data: the interview data was coded and classified according to complexity factors with the help of AtlasTi programme. A parallel coder was used to confirm the reliability of coding. After parallel coding the parameters of classification were redefined. The variables of the questionnaires were dealt with using the SPSS programme.

After complexity factor coding, each of six factors was examined separately.

\section{Results - Manifestation of Complexity Factors and Complexity-Related Workload Factors}

Geographical dispersion, i.e. sphere of operations. Both groups did multi-locational work. Global groups worked at home, at the primary workplace, in means of transportation, at secondary places i.e. secondary places of their own company or places belonging to customers and at third places i.e. restaurants, hotel rooms and other places normally associated with free time or leisure time as shown in Table 1. Of the locally mobile group only the maintenance men worked from home on a regular basis. The home was used for planning and organizing work for the next day. Security personnel and community nurses rarely worked at home and they did not construct a virtual connection for a work from home basis as shown in Table 1.

As expected, the geographical dispersion of working places was greatest with those moving globally. Representatives of small and large companies operating globally worked on different continents, and another group traveled within Europe. Locally mobile groups worked in a local area, which varied in size from tens to hundreds of square kilometers. While the globally mobile stayed at least a day in their target area, the locally mobile groups visited several places during their working day.

Table 1 also presents the devices used for constructing virtual space of different workplaces. After that, there is a condensed description of the reasons for using virtual working spaces. Geographical dispersion of the team, multi-locational work as well as the mobility of employee were the reasons for constructing and using the virtual work environment.

The workload factors related to dispersed and multi-locational work were both mental and social in nature (table 2). However, the main cause lay in the communication connections. Challenging duties and demanding human relationships associated with poorly or roughly working virtual connections were a source of stress. The malfunctioning of communication connections, devices or applications at secondary and third workplaces posed even experiences of isolation and loneliness. 


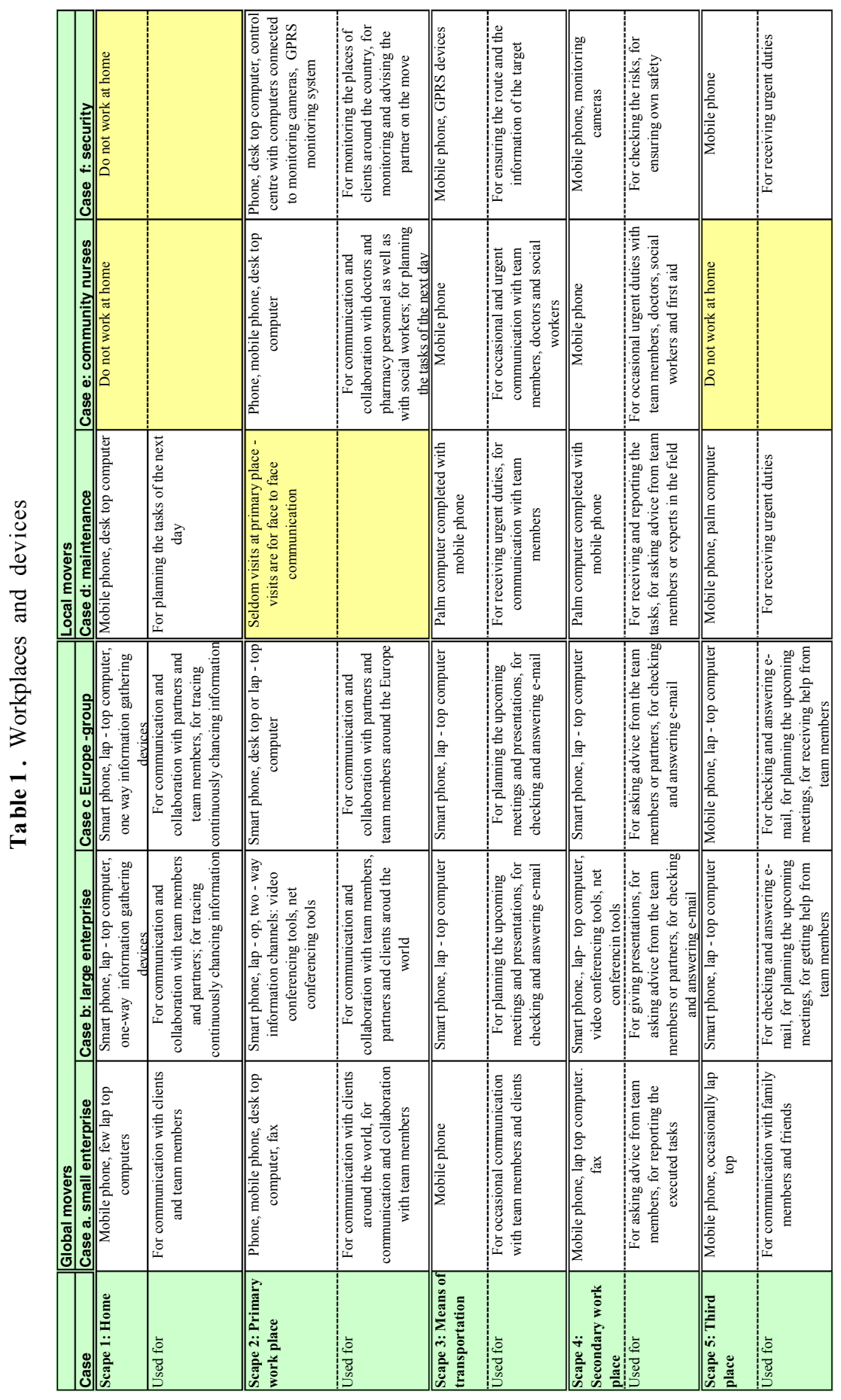


An integral part of the feeling of well-being was the support one was able to receive through well-functioning channels of communication and collaboration. Good connectivity potential was the "thing" that interviewed mobile employees hoped for and appreciated.

Mobility. Comparison of locally and globally mobile groups showed that local groups considered themselves to be more mobile than global groups. This was due to the frequency of trips: locally mobile groups were continuously on the move during their everyday work. Global movers were traveling for several days during the month but they also stayed in the main workplace for longer periods than the locally mobile. While moving, both groups used ICT tools, mainly mobile phones for communication.

The described workload factors related to the mobility of employee were mainly physical in nature. From the viewpoint of the virtual working environment the quality of the mobile device was emphasized. Because the virtual working environment constructing ICT devices were kept while moving in a pocket or in a backpack, they were expected to be light and tiny. However, problems of visibility emerged when devices were smaller. For example, the maintenance men moved in dark wells and engine rooms and had visual difficulties with the palm computer they used. This dilemmatic question between size and visual requirements as well as portability requirements (table 2) is mainly the concern of the microergonomics discipline.

Cultural diversity. Since the basis of mobile work was to meet clients face-to-face, mobile employees encountered a multitude of different individuals. In particular, the cultural diversity of actors in the globally mobile groups was a complexity factor both in physical and virtual work spaces. The workload factor caused by the diversity of actors was both mental and social.

Time. There were great differences in what employees comprehended as asynchronous work. Representatives of the global company had experiences of asynchronous time having worked in different time zones. Locally mobile employees determined the dissimilarity in working hours as asynchronous working. For example, the security personnel, who predominantly worked at night time, considered their work asynchronous. Asynchronous working increased the need for coordinating one's use of time. In this study, especially in the groups working globally, asynchronous working had the effect of changing the hours and rhythm of the work. Employees did not have uninterrupted working days starting at a particular time and ending at another, but instead altered rhythms of their days as well as their weeks according to the demands of their tasks. Working periods could take place early in the morning, in the afternoon and in the evening. Work might be done to some extent every day of the week. Asynchrony with using a virtual work space caused physical, mental and social strain (table 2). For example, in planning a net meeting it had to be taken into account that members were spread out around the world. This caused an inclination towards unconventional working hours with evening and weekend work. Also the need to be constantly available, affected the experiences of strain. Based on the survey study (VTQ) there was statistically significant $(\mathrm{p}<.001)$ difference in weekly working hours 
between globally and those locally mobile. The globally mobile worked on average ten hours more per week $(\mathrm{m}=49 \mathrm{~h} /$ week $)$ when compared to the locally mobile employees $(\mathrm{m}=39 \mathrm{~h} /$ week $)$.

Temporarity. When evaluating the permanence of working groups and their collaboration, i.e. the time frame of a project and existence of the team, it became evident that members of large global companies and the Europe group performed more project work than other groups. According to the interviews, the temporary nature of the projects was considered both as a complexity factor and a workload factor. It was hard to forge good and trustworthiness relationships, if the team or partners in cooperation constantly changed. The workload can be defined as mental and social. The main workload factor was related to too many and constantly changing human relations.

The workload factors associated with the complexity factors are summarized in Table 2. The complexity factors interrelated with different types of workload components, i.e. physical, mental and social and, furthermore, they induced distinct workload factors. To reduce the manifestation of the workload factors and to enhance well-being, fundamental requirements for the virtual environment can be presented.

Table 2. Complexity factors of mobile work related to workload factors and requirements for virtual environment

\begin{tabular}{||c|c|c|c||}
\hline Complexity factor & Workload & $\begin{array}{c}\text { Distinct Workload } \\
\text { factors }\end{array}$ & $\begin{array}{c}\text { Requirements for } \\
\text { well-being in virtual } \\
\text { environment }\end{array}$ \\
\hline \hline $\begin{array}{c}\text { Geographical } \\
\text { dispersion }\end{array}$ & Mental and social & Bad connections & Connectivity \\
\hline $\begin{array}{c}\text { Mobility } \\
\text { Diversity of actors }\end{array}$ & Mental and social & $\begin{array}{c}\text { Cultural diversity, } \\
\text { demanding human } \\
\text { relations }\end{array}$ & Portability \\
\hline Asynchronous work \\
time
\end{tabular}


Mediated interaction. As the degree of mobility and the geographical dispersion of the workplaces increased so did the demands for mediated interaction, i.e. the use of the virtual working spaces. The workload experiences of the interviewee were associated with the interpretation of messages sent with a means of communication and collaboration: mediated interaction carried many opportunities for misunderstandings, which could even be fateful, for example, in the work of security personnel during dangerous situations the supervisor in the guarding centre directs the movement of a guard in the field. The explicit and shared understanding of messages between them was essential. On that account of this the workload factors related to mediated interaction were mainly mental and social in nature (table 2). The central issue can be consilidated to the question of cognitive abilities, i.e. how successfully one can build up mental and social constructions in virtual working environments.

\section{Discussion}

Figure 2 shows a systemic generalization of findings concerning the complexity factors of the virtual environment and the possible dissection levels of requirements for virtual communication and collaboration. The levels are presented in a protocol manner (comparable to Internet protocol) to underline the layered nature of the virtual environment, as spatial mobility refers not only to extensive moving of people, but also the global flux of objects, symbols, and space itself [8]. As such it evokes complex patterns of human interaction. When mediated interaction is compared to the face-to-face communication, it is much more layered and therefore susceptible to disturbances and breaks. The employee has to cope with different cognitive skill requirements as well as with the cultural diversity of actors and colleagues when working in a virtual manner. Disturbances at this level lead easily to misunderstandings. Coping with the diversity of actors in mediated interaction environments is not only a complexity factor but also a mental and social workload factor.

In particular, these upper cognitive levels of the virtual working environment are far too less known $[1,4,7]$. Important research topics are expected to be related to questions of decision-making in demanding tasks in virtual environments. This is one of the forthcoming focuses of, for example, macroergonomic research topics. There is an evident need to develop tools both for managers and virtual environment designers to better control the questions related to this cognitive virtual reality.

Dropouts in virtually mediated communication can also be encountered due to time constraints, i.e. asynchronous work. Time constraints and time asynchrony are also questions that place stress on developing both work management styles and the functioning of devices and applications. More effective control of time is both a micro- and macroergonomic concern.

The minimum level required for successful communication and collaboration in virtual environment concerns proper functioning of the connections, devices and applications. The flow of communication and collaboration breaks if this physical level of connections, devices or applications does not work or exist. The inoperative ICT tools could be fatal due to misunderstanding at the upper layers of the virtual environment. In mobile work, there are additional requirements concerning the 
devices. The transfer to virtual working space via devices and applications must be attained regardless of the time and place. The operational environment of a mobile employee should be portable as well as easily perceivable.

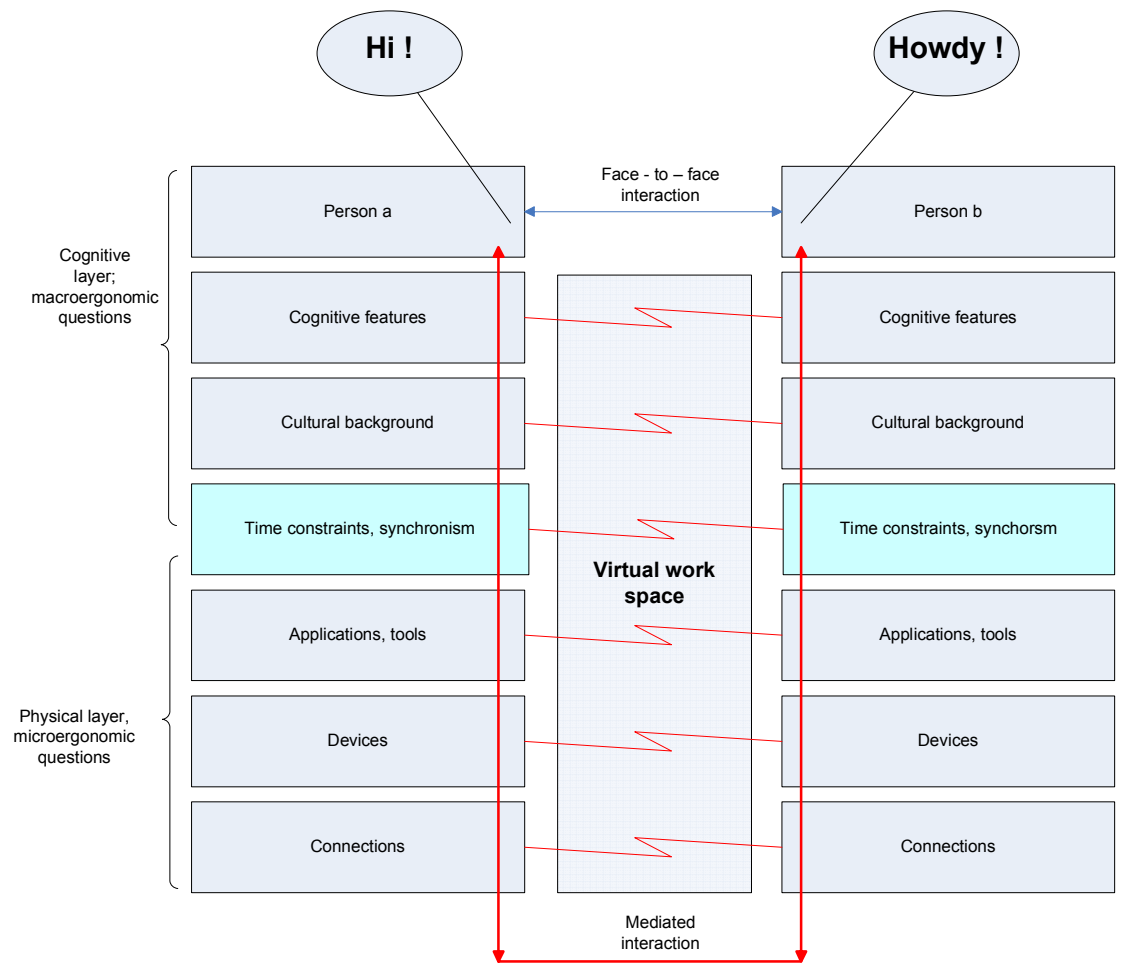

Fig. 2. Complexity of communication and collaboration in mobile and virtual work

In conclusion, with the complexity and workload factors of the virtual environment of mobile work, the question at the lower levels lies in the transfer capability of communication. Compared to this at the upper levels, the question is in the semantic transfer of the message. Although we have the technological capability to work across time and distance, the fact is that we need new competencies and practices to do these things. Working in mobile virtual teams requires much more than computers and technology.

\section{References}

1. Andriessen, J.H.E.: Working with groupware. In: Understanding and evaluating collaboration technology, Springer, Berlin Heidelberg New York (2003)

2. Andriessen, J.H.E., Vartiainen, M. (eds.): Mobile Virtual Work. A New Paradigm? Springer, Berlin Heidelberg New York (2006) 
3. Cascio, W.F.: Managing a virtual workplace. Academy of Management Executive 14(3), 81-90 (2000)

4. Corso, M., Martini, A., Pellegrini,: Knowledge sharing in Mobile Work. In: Corso, M., Martini, A. (eds.) Mobile Virtual Work. A New Paradigm? pp. 45-69. Springer, Heidelberg (2006)

5. Duarte, D.I., Snyder, N.T: Mastering Virtual Teams. In: Strategies, tools and techniques that succeed, Jossey-Bass, San Francisco (2001)

6. Gareis, K., Lilischkis, S., Mentrup, A.: Mapping the Mobile eWorkforce in Europe. In: Andriessen, J.H.E., Vartiainen, M. (eds.) Mobile Virtual Work. A New Paradigm? pp. 4569. Springer, Berlin Heidelberg New York (2006)

7. Grabowski, M., Roberts, K.H.: Risk Mitigation in Virtual Organizations. Organization Science : Communication Processes for Virtual Organizations, vol. 10(6), pp. 704-721 (1999)

8. Kakihara, M., Sørensen, C.: Expanding the "mobility" concept. Siggroup Bulletin 22(3), 33-37 (2001)

9. Kakihara, M., Sørensen, C.: Practicing Mobile Professional Work: Tales of Locational, Operational and Interactional Mobility. INFO: The. Journal of Policy, Regulation and Strategy for Telecommunication, Information and Media 6, 180-187 (2004)

10. Kokko, N., Vartiainen, M., Hakonen, M.: Work Stressors on Virtual Organizations. In: ITA Conference 2004, Greece. Crete (September 6-9, 2004)

11. Nonaka, I., Takeuchi, H.: The knowledge-creating company. In: How Japanese companies create the dynamics of innovation, Oxford University Press, New York (1995)

12. Lazarus, R.S.: Psychological stress in workplace. Handbook on Job Stress, Journal of Social Behaviour and Personality 6, 1-13 (2004)

13. Richter, P., Meyer, J., Sommer, F.: Well-being and Stress in Mobile and Virtual Work. In: Andriessen, J.H.E., Vartiainen, M. (eds.) Mobile Virtual Work: A New Paradigm? pp. 4569. Springer, Heidelberg (2006)

14. Wilmar, B., Schaufeli, W.B., Salanova, M., González-Romá, V., Bakker, A.B.: The Measurement of Engagement and Burnout: A Two Sample Confirmatory Factor Analytic Approach. Journal of Happiness Studies 3(1), 71-92 (2002)

15. Vartiainen. , M.: Mobile Virtual Work - Concepts, Outcomes and Challenges. In: Andriessen, J.H.E., Vartiainen, M. (eds.) Mobile Virtual Work. A New Paradigm? pp. 1344. Springer, Berlin Heidelberg New York (2006)

16. Yin, R.K.: Case Study Research: design and Methods. In: Applied Social Research Methods Series, 3rd edn., vol. 5, Sage Publications, Thousand Oaks, California (2003) 